\title{
Expression patterns of STAT3, ERK and estrogen-receptor a are associated with development and histologic severity of hepatic steatosis: a retrospective study
}

Euno Choi ${ }^{1 \dagger}$, Won Kim² ${ }^{2 \dagger}$, Sae Kyung Joo², Sunyoung Park', Jeong Hwan Park', Yun Kyung Kang ${ }^{3}$, So-Young $\mathrm{Jin}^{4}$ and Mee Soo Chang ${ }^{1 *}$

\begin{abstract}
Background: Hepatic steatosis renders hepatocytes vulnerable to injury, resulting in the progression of preexisting liver disease. Previous animal and cell culture studies implicated mammalian target of rapamycin (mTOR), signal transducer and activator of transcription-3 (STAT3), extracellular signal-regulated kinase (ERK) and estrogen-receptor $a$ in the pathogenesis of hepatic steatosis and disease progression. However, to date there have been few studies performed using human liver tissue to study hepatic steatosis. We examined the expression patterns of mTOR, STAT3, ERK and estrogen-receptor a in liver tissues from patients diagnosed with hepatic steatosis.

Methods: We reviewed the clinical and histomorphological features of 29 patients diagnosed with hepatic steatosis: 18 with non-alcoholic fatty liver disease (NAFLD), 11 with alcoholic fatty acid disease (AFLD), and a control group (16 biliary cysts and 22 hepatolithiasis). Immunohistochemistry was performed on liver tissue using an automated immunostainer. The histologic severity of hepatic steatosis was evaluated by assessing four key histomorphologic parameters common to NAFLD and AFLD: steatosis, lobular inflammation, ballooning degeneration and fibrosis.

Results: mTOR, phosphorylated STAT3, phosphorylated pERK, estrogen-receptor a were found to be more frequently expressed in the hepatic steatosis group than in the control group. Specifically, mTOR was expressed in 78\% of hepatocytes, and ERK in 100\% of hepatic stellate cells, respectively, in patients with NAFLD. Interestingly, estrogenreceptor a was diffusely expressed in hepatocytes in all NALFD cases. Phosphorylated (active) STAT3 was expressed in $73 \%$ of hepatocytes and $45 \%$ of hepatic stellate cells in patients with AFLD, and phosphorylated (active) ERK was expressed in hepatic stellate cells in all AFLD cases. Estrogen-receptor a was expressed in all AFLD cases (focally in $64 \%$ of AFLD cases, and diffusely in 36\%). Phosphorylated STAT3 expression in hepatocytes and hepatic stellate cells correlated with severe lobular inflammation, severe ballooning degeneration and advanced fibrosis, whereas diffusely expressed estrogen-receptor a correlated with a mild stage of fibrosis.
\end{abstract}

Conclusions: Our data indicate ERK activation and estrogen-receptor a may be relevant in the development of hepatic steatosis. However, diffuse expression of estrogen-receptor a would appear to impede disease progression, including hepatic fibrosis. Finally, phosphorylated STAT3 may also contribute to disease progression.

Keywords: Hepatic steatosis, Non-alcoholic, Alcoholic, mTOR, pSTAT3, pERK, Estrogen-receptor a

\footnotetext{
*Correspondence: meesooch@snu.ac.kr

${ }^{\dagger}$ Equal contributors

'Department of Pathology, Seoul National University Boramae Hospital, Seoul

National University College of Medicine, 20 Boramae-ro 5-gil, Dongjak-gu,

Seoul 07061, Korea

Full list of author information is available at the end of the article
} 


\section{Background}

Hepatic steatosis is a frequent histological finding in liver biopsy specimens. The causes of hepatic steatosis include obesity, excessive alcohol intake, chronic autoimmune diseases, Wilson's disease, hepatitis $\mathrm{C}$ virus and certain pharmacological drugs [1]. Increasing evidence indicates that hepatic steatosis enhances hepatocellular susceptibility to additional injuries, eventually leading to hepatic fibrosis [2]. The molecular factors contributing to hepatic steatosis, hepatocellular damage, and hepatic fibrosis have been studied, but their action mechanisms are still not fully resolved.

The mammalian target of rapamycin (mTOR) controls lipid biosynthesis via various effector molecules, such as sterol regulatory element-binding protein like-1c (SREBP-1c) [3] which engages in the development of hepatic steatosis [4]. mTOR, signal transducer and activator of transcription-3 (STAT3), and extracellular signal-regulated kinase (ERK) all belong to downstream mediators of leptin signaling [5-7], and it has been shown that leptin plays a crucial role in hepatic lipid regulation [8]. In cell experiments, signal transducer and activator of transcription (STAT3) has been shown to be required for IL-6-mediated activation of hepatic stellate cells, eventually resulting in hepatic fibrosis [9]. Also, the kinases, including extracellular signal-regulated kinase (ERK), are activated early in liver injury, and then led to hepatic fibrosis of rats [10]. In addition, estrogen is a steroid hormone that preserves hepatic lipid hemostasis by acting via estrogen-receptor $\alpha$ $[11,12]$. Animal and cell culture studies suggest estrogen prevents hepatic fibrosis by blocking lipid peroxidation and production of reactive oxygen species [13].

To date, the cellular localization and expression patterns for leptin signaling proteins and estrogen-receptor $\alpha$ have not been collectively determined in liver tissue, and specifically in hepatocytes and hepatic stellate cells. Hepatic stellate cells line the perisinusoidal space, and are located between hepatocytes and the sinusoidal endothelium [14]. When activated by liver injury, these cells newly express $\alpha$-smooth muscle actin and transdifferentiate into myofibroblast-like cells, which form the extracellular matrix that leads to hepatic fibrosis [14-16].

Non-alcoholic fatty liver disease (NAFLD) and alcoholic fatty liver disease (AFLD) are two common causes of chronic liver disease worldwide [17]. Although managed differently [18], NAFLD and AFLD possess similar histomorphologic features (e.g., macrovesicular steatosis, lobular inflammation, and hepatocyte ballooning degeneration) [19-21] and a similar course of disease progression (simple steatosis followed by steatohepatitis, fibrosis, and micronodular cirrhosis) [21-23]. They have shared and disease-specific mechanisms of lipid accumulation, and their pathogenesis includes oxidative stress, iron deposition, overexpression of cytochrome P450E1, and involvement of endotoxins and tumor necrosis factor $\alpha$ [19-21]. Oxidative stress induces expression of lipid metabolism-associated transcription factors (e.g., SREBP-1c) that regulate de novo fatty acid synthesis [4]. Diseasespecific mechanisms include augmented lipolysis in adipose tissue and consequent increases in circulating free fatty acid levels in NAFLD, but not in AFLD [20]. The major substrate of cytochrome P450E1 is excessive free fatty acids in NAFLD and ethanol in AFLD [4, 19, 24].

Here, we evaluated the clinical and histomorphologic features and expression patterns of mTOR, phosphorylated STAT3 (pSTAT3), phosphorylated ERK (pERK) and estrogen-receptor $\alpha$ in hepatocytes and hepatic stellate cells in liver tissue from patients with hepatic steatosis, along with a control group (biliary cysts and hepatolithiasis). Our goal was to determine the roles these proteins might play in the development of hepatic steatosis.

\section{Methods}

\section{Patients and liver tissues}

This retrospective study comprised 29 patients with hepatic steatosis; 18 diagnosed with NAFLD and 11 diagnosed with AFLD. Patients were selected from the electronic database of the Department of Pathology, Seoul National University Boramae Hospital. Information in the database was obtained from needle biopsies of liver tissue during 2005-2011. Diagnoses were based on clinicopathological correlations, the results of a serologic test of liver function, causes of the liver dysfunction, a history of alcohol intake, and histomorphologic evaluation of liver tissue biopsies $[25,26]$. Inclusion criterion for liver biopsies was a tissue specimen $>1 \mathrm{~cm}$ in length without fragmentation or $>$ 10 portal tracts with macrovesicular steatosis in $>5 \%$ of hepatocytes.

Results of a serologic test of liver function were regarded as abnormal if aspartate transaminase (AST) or alanine transaminase (ALT) levels were each $>40 \mathrm{U} / \mathrm{L}$ and total bilirubin levels were $>1.2 \mathrm{mg} / \mathrm{dL}$. Body mass index (BMI) was determined as body weight in kilograms divided by height in meters squared. According to the revised World Health Organization criteria for obesity in the Asia Pacific region, BMIs > 25, 23-24.9, 18.5-22.9, and $<18.5$ correspond to obesity, overweight, normal heathy weight, and underweight, respectively [27]. Alcoholic fatty liver disease was considered if alcohol consumption was excessive (>30 g/day for men and > $20 \mathrm{~g} /$ day for women) as per each patient's self-reporting and interviews with family members.

To better understand protein expression patterns in non-neoplastic hepatocytes, we used a control group composed of surgically resected liver tissue from 16 cases of biliary cysts and 22 cases of hepatolithiasis (without steatosis, fibrosis or dysplasia). 


\section{Histomorphologic assessment}

Formalin-fixed, paraffin-embedded (FFPE) tissue samples were microsectioned and stained with both hematoxylin and eosin and Masson's trichrome stain. Diagnoses of NAFLD and AFLD were based on clinical features and pathological criteria [23, 25, 26]. Common histomorphologic features of NAFLD and AFLD include the four core features of steatosis, lobular inflammation, ballooning degeneration, and fibrosis, all of which were assessed using the Nonalcoholic Steatohepatitis Clinical Research Network (NASH CRN) NAFLD activity score (NAS) system [26] and the descriptive system proposed for alcoholic liver disease [23]. NAFLD was also sub-classified as significant or mild using the steatosis, activity, and fibrosis (SAF) scoring system [28]. The SAF and NASH CRN NAS systems apply similar criteria when determining grades and scores for steatosis, activity and fibrosis. The main difference is the evaluation criteria of ballooning degeneration; SAF system puts the size and number of the enlarged cells into consideration while the NASH CRN system considers only the number of enlarged cells [29].

\section{Immunohistochemistry}

Immunohistochemistry was performed using an automated Ventana Benchmark XT immunostainer (Ventana BenchMark XT; Ventana Medical Systems Inc., Tucson, AZ, USA) according to the manufacturer's protocol. Briefly, 3- $\mu \mathrm{m}$-thick tissue sections were placed on electrostatic charged glass slides, deparaffinized, and subjected to antigen retrieval. The antigen was detected using ultraView Universal DAB Detection Kit (Ventana Medical Systems Inc.). For double staining, an ultraView Universal Alkaline Phosphatase Red Detection Kit (Ventana Medical Systems Inc.) was used. The following primary antibodies were used at the following dilutions: mTOR (49F9, 1:50; Cell Signaling, Danvers, MA, USA), pSTAT3 (1:50; Cell Signaling), pERK1/2 (44/42 MAPK, Thr 202/Tyr 204) (E10, 1:50; Cell Signaling), estrogen-receptor $\alpha$ (SP1, ready to use; Ventana Medical Systems Inc.), and $\alpha$-smooth muscle actin (1A4, 1:500; Labvision, Fremont, CA, USA). For negative controls, each antibody was replaced with phosphate-buffered saline.

For mTOR and the phosphorylated (i.e., active) forms of STAT3 and ERK, nuclear staining was evaluated and considered positive when $\geq 10 \%$ of hepatocytes, or any of the hepatic stellate cells, were stained. For estrogen-receptor $\alpha$ expression, the area of positively stained hepatic nuclei was categorized as $1(<10 \%), 2(10-50 \%)$, or $3(>50 \%)$, and staining intensity was graded as 1 (weak), 2 (moderate), or 3 (strong). A total score (1 to 9 ) was calculated by multiplying area and intensity scores. Estrogen-receptor $\alpha$ expression was also defined as negative, focal, or diffuse (scores of 1 , $2-4$, and $6-9$, respectively). $\alpha$-smooth muscle actin was used as a marker for hepatic stellate cells $[13,14]$.

\section{Statistical analysis}

Statistical differences were analyzed using the chi-square test or Fisher's exact test (2-sided) for categorical variables, and the Mann-Whitney $U$ test or analysis of variance for continuous variables. $P$ values $<0.05$ were considered statistically significant. Statistical analyses were performed using SPSS Statistics version 20.0 (IBM Inc., Armonk, NY, USA).

\section{Results}

Clinical and histomorphologic features of hepatic steatosis

Clinical and histomorphologic characteristics of patients with hepatic steatosis and a control group (biliary cysts and hepatolithiasis cases without steatosis, fibrosis or dysplasia) are summarized in Table 1. Most NAFLD patients were obese, whereas $55 \%$ of AFLD patients were of normal weight $(P=0.001)$. An AST/ALT ratio $\leq$ 2 was more often observed in the NAFLD group than the AFLD group, but the difference was not statistically significant. The NAFLD group showed milder histomorphologic features in lobular inflammation, ballooning degeneration and fibrosis than the AFLD group $(P<0.05$, respectively). Representative histomorphologic features are shown in Fig. 1.

\section{Protein expression patterns in hepatic steatosis}

The hepatic steatosis group frequently showed more positive expression of proteins than the control group $(P<0.05$ for the most proteins). The only exception to this trend was pSTAT3 in hepatic stellate cells which showed marginal expression differences $(P=0.055)$ (Table 2$)$. In detail, hepatic mTOR was expressed in $78 \%$ of NAFLD cases. Of interest, estrogen-receptor $\alpha$ expression was diffusely positive in all NAFLD cases. NAFLD cases were also characterized by low (hepatocytes, $17 \%$ ) or no (hepatic stellate cells) pSTAT3 expression, and low (hepatocytes, 22\%) or predominant (hepatic stellate cells, 100\%) pERK expression. Notably, in AFLD, pSTAT3 was often observed in both hepatocytes (73\%) and hepatic stellate cells (45\%). All AFLD cases had estrogen-receptor $\alpha$-positive hepatocytes, with focal positivity in $64 \%$ of cases and diffuse positivity in $36 \%$. Estrogen-receptor $\alpha$ was not sufficiently expressed to be considered positive by immunohistochemical evaluation in the non-neoplastic hepatocytes of the control group (biliary cyst and hepatolithiasis cases without hepatic steatosis, fibrosis or dysplasia). Representative immunohistochemical features can be seen in Fig. 2. We observed non-specific cytoplasmic or membranous staining of mTOR in non-neoplastic hepatocytes in all hepatic steatosis and control cases. Hence, only mTOR staining in the nucleus was evaluated. 
Table 1 Clinicopathologic and histomorphologic features in hepatic steatosis patients

\begin{tabular}{|c|c|c|c|}
\hline & $\begin{array}{l}\text { NAFLD } \\
(n=18)\end{array}$ & $\begin{array}{l}\text { AFLD } \\
(n=11)\end{array}$ & $\begin{array}{l}\text { Control } \\
(n=38)\end{array}$ \\
\hline \multicolumn{4}{|l|}{ Clinical Features } \\
\hline Age, mean (range) years & $23 \pm 6.4(11-36)^{a}$ & $41 \pm 9.5(23-59)^{a}$ & $61 \pm 12.5(34-89)^{\mathrm{a}}$ \\
\hline \multicolumn{4}{|l|}{ Sex } \\
\hline male & $15(83 \%)$ & $9(82 \%)$ & $11(29 \%)$ \\
\hline female & $3(17 \%)$ & $2(18 \%)$ & $27(71 \%)$ \\
\hline \multicolumn{4}{|l|}{ BMI } \\
\hline underweight & 0 & $1(9 \%)$ & $2(5 \%)$ \\
\hline normal & $3(17 \%)$ & $6(55 \%)$ & $13(34 \%)$ \\
\hline overweight & 0 & $2(18 \%)$ & $15(39 \%)$ \\
\hline obese & $15(83 \%)$ & $2(18 \%)$ & $8(21 \%)$ \\
\hline \multicolumn{4}{|l|}{ Liver Biochemistry } \\
\hline \multicolumn{4}{|l|}{ AST/ALT ratio } \\
\hline$\leq 2$ & $14(78 \%)$ & $6(55 \%)$ & - \\
\hline$>2$ & $4(22 \%)$ & $5(45 \%)$ & - \\
\hline AST, median (mean, range) U/L & $79(67,11 \sim 192)$ & $67(105,21 \sim 152)$ & $22(35,12 \sim 249)$ \\
\hline ALT, median (mean, range) U/L & $84(124,11 \sim 384)$ & $44(35,12 \sim 204)$ & $17(31,7 \sim 252)$ \\
\hline $\mathrm{TB}$, median (mean, range) mg/dL & $0.8(0.9,0.5 \sim 12.4)$ & $14(9,0.7 \sim 19.8)$ & $0.7(0.8,0.3 \sim 3.5)$ \\
\hline \multicolumn{4}{|l|}{ Histomorphological Features } \\
\hline Steatosis & & & None \\
\hline $5-33 \%$ & $4(22 \%)$ & $2(18 \%)$ & \\
\hline$>33-66 \%$ & $5(28 \%)$ & $5(45 \%)$ & \\
\hline$>66 \%$ & $9(50 \%)$ & $4(36 \%)$ & \\
\hline \multicolumn{2}{|c|}{ Lobular inflammation (inflammatory foci/200× field) } & & None \\
\hline$<2$ & $16(89 \%)$ & $6(55 \%)$ & \\
\hline $2-4$ & $2(11 \%)$ & $3(27 \%)$ & \\
\hline$>4$ & 0 & $2(18 \%)$ & \\
\hline \multicolumn{2}{|l|}{ Ballooning degeneration } & & None \\
\hline none & $9(50 \%)$ & $1(9 \%)$ & \\
\hline few & $8(44 \%)$ & $5(45 \%)$ & \\
\hline many & $1(6 \%)$ & $5(45 \%)$ & \\
\hline \multicolumn{2}{|l|}{ Fibrosis } & & None \\
\hline none & $6(33 \%)$ & $1(9 \%)$ & \\
\hline perisinusoidal or peripota1 & $11(61 \%)$ & $1(9 \%)$ & \\
\hline Perisinusoidal \& potal/periportal & $1(6 \%)$ & $1(9 \%)$ & \\
\hline bridging fibrosis & 0 & $3(27 \%)$ & \\
\hline cirrhosis & 0 & $5(45 \%)$ & \\
\hline
\end{tabular}

NAFLD non-alcoholic fatty liver disease

AFLD alcoholic fatty liver disease

Control, 16 cases of biliary cyst and 22 cases of hepatolithiasis

${ }^{a}$ mean \pm standard deviation

AST aspartate transaminase

$A L T$ alanine transaminase

$T B$ total bilirubin 


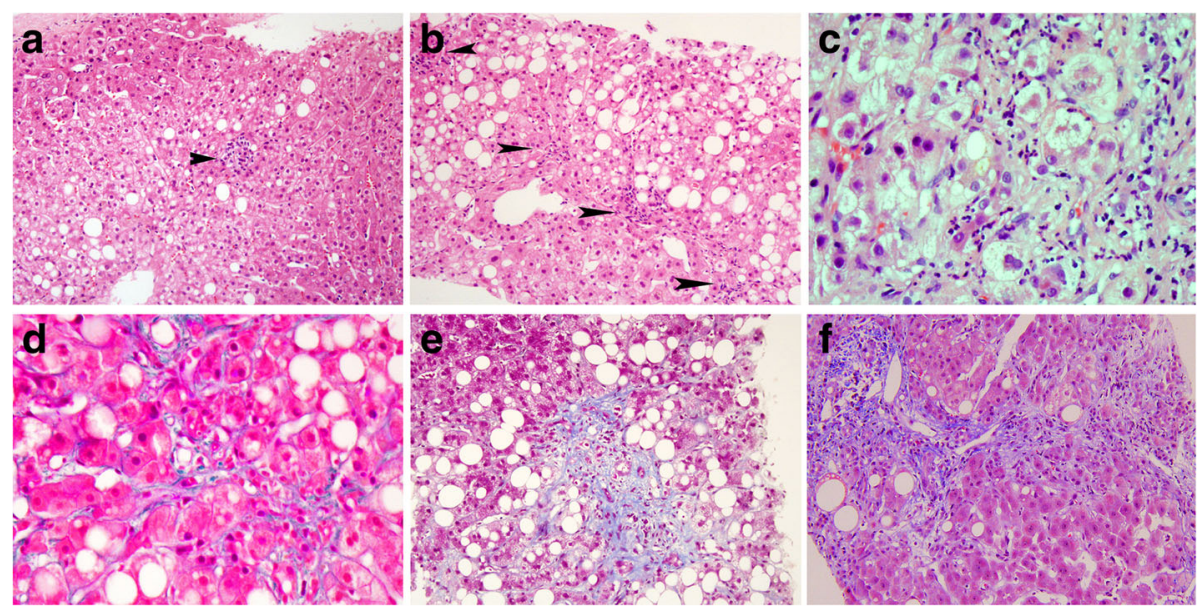

Fig. 1 Representative histomorphologic features of hepatic steatosis. (a) Lobular inflammation, with a single focus of inflammatory cells (arrow) (H\&E). (b) Lobular inflammation, with four foci of inflammatory cells (arrows) (H\&E). (c) Ballooning degeneration, with many enlarged hepatocytes including at least one twice the size of a normal cell (H\&E). (d) Perisinusoidal fibrosis (Masson's trichrome). (e) Periportal fibrosis (Masson's trichrome). (f) Septal fibrosis (Masson's trichrome)

\section{Relationship between protein expression and histologic severity in hepatic steatosis}

Expression of pSTAT3 in hepatocytes and hepatic stellate cells significantly correlated with severe histologic features (e.g., severe lobular inflammation, severe ballooning inflammation, and advanced stage of fibrosis; $P<0.05$, respectively) (Table 3). In contrast, diffuse nuclear expression of estrogen-receptor $\alpha$ in hepatocytes correlated with mild histologic features $(P<0.05)$. There was no statistical relationship between pERK expression and histologic severity, although there was a near significant relationship $(P=$
0.083) between pERK expression and lobular inflammation in hepatic stellate cells.

Using the NASH CRN and SAF histologic scoring systems, we classified NAFLD as mild, severe, or significant. Ballooning degeneration and lobular inflammation were significantly increased in severity in patients with either severe or significant NAFLD when compared to those with mild NAFLD $(P<0.05$, respectively) (Additional file 1$)$. However, it should be noted that there was no difference in protein expression between the severe/significant subgroup and the mild subgroup.

Table 2 Comparison of protein expression features in hepatic steatosis patients and the control group

\begin{tabular}{|c|c|c|c|c|c|}
\hline & Total hepatic steatosis & NAFLD & AFLD & Control & ${ }^{*} P$ value \\
\hline & $(N=29)$ & $(n=18)$ & $(n=11)$ & $(N=38)$ & \\
\hline \multicolumn{6}{|l|}{ Protein expression in hepatocytes } \\
\hline mTOR, positive & $16(55 \%)$ & $14(78 \%)$ & $2(18 \%)$ & $1(3 \%)$ & $<0.001$ \\
\hline pSTAT3, positive & $11(38 \%)$ & $3(17 \%)$ & $8(73 \%)$ & $4(11 \%)$ & 0.007 \\
\hline pERK, positive & $8(28 \%)$ & $4(22 \%)$ & $4(36 \%)$ & $1(3 \%)$ & 0.003 \\
\hline Estrogen-receptor a, positive & $29(100 \%)$ & $18(100 \%)$ & $11(100 \%)$ & 0 & $<0.001$ \\
\hline Focal positive & $7(24 \%)$ & 0 & $7(64 \%)$ & 0 & \\
\hline Diffuse positive & $22(76 \%)$ & $18(100 \%)$ & $4(36 \%)$ & 0 & \\
\hline \multicolumn{6}{|c|}{ Protein expression in hepatic stellate cells } \\
\hline pSTAT3, positive & $5(17 \%)$ & 0 & $5(45 \%)$ & $2(5 \%)$ & 0.055 \\
\hline pERK, positive & $29(100 \%)$ & $18(100 \%)$ & $11(100 \%)$ & $3(8 \%)$ & $<0.001$ \\
\hline
\end{tabular}

NAFLD non-alcoholic fatty liver disease

AFLD alcoholic fatty liver disease

Control, non-neoplastic hepatosytes from 16 cases of biliary cyst and 22 cases of hepatolithiasis

${ }^{*} P$ value between total hepatic steatosis group $(N=29)$ and the control group $(N=38)$ 


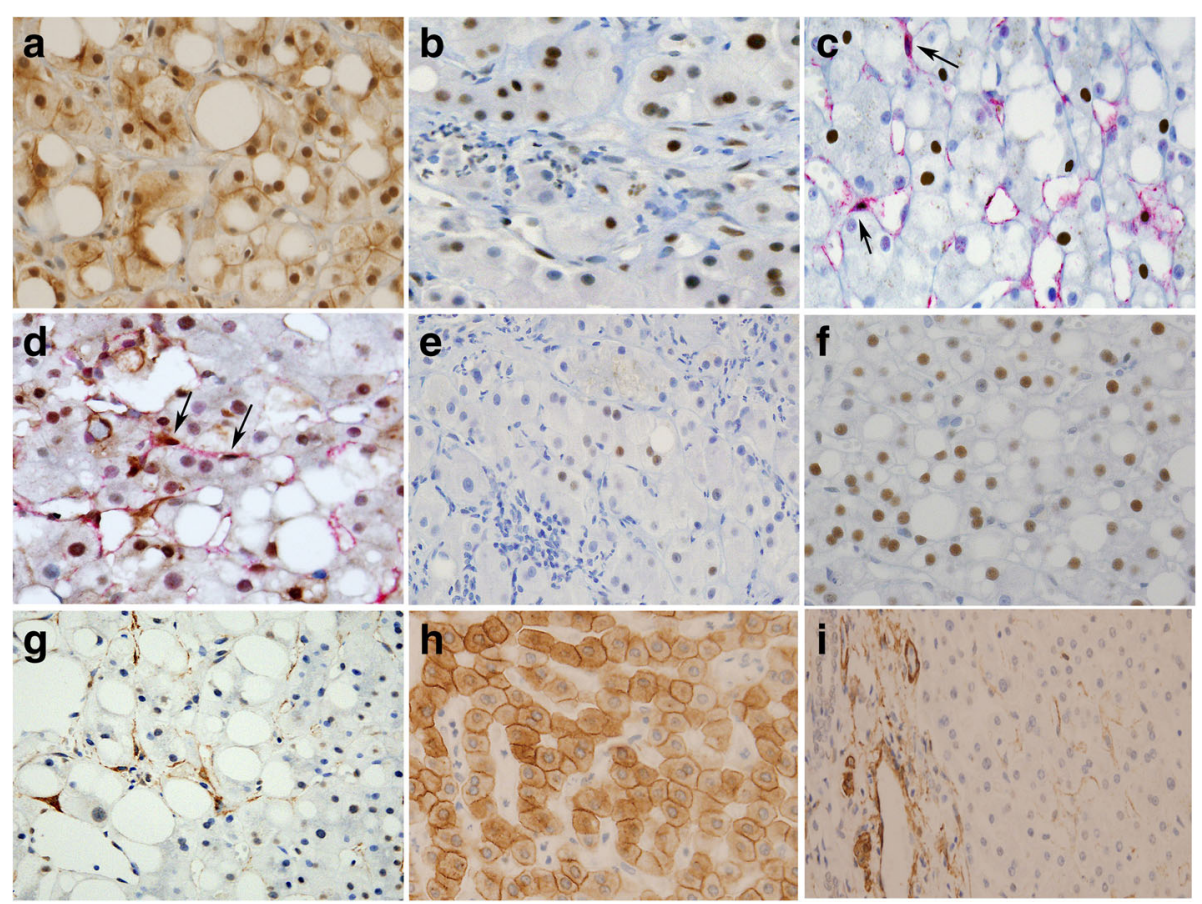

Fig. 2 Representative immunohistochemical features in hepatic steatosis (a-g) and the control group (h-i). (a) Nuclear and/or weak cytoplasmic and membranous expression of mammalian target of rapamycin (mTOR). (b) Nuclear staining of phosphorylated signal transducer and activator of transcription-3 (pSTAT3) in hepatocytes. (c) Double staining of pSTAT3 (nucleus) and a-smooth muscle actin (cytoplasm, red color) in hepatic stellate cells (arrows). (d) Double staining of extracellular signal-regulated kinase (pERK) (nucleus) and a-smooth muscle actin (cytoplasm, red color) in hepatic stellate cells (arrows). (e) Focal expression of estrogen-receptor a in hepatocyte nuclei. (f) Diffuse expression of estrogen-receptor a. (g) a-smooth muscle actin-positive cytoplasm in hepatic stellate cells. (h) Cytoplasmic and membranous staining of mTOR in non-neoplastic hepatocytes in a control sample. (i) Inconspicuous staining of a-smooth muscle actin (inactive hepatic stellate cells; center of figure) in a control sample, while strong positive staining in vessel walls as an internal positive control (left side of figure)

Table 3 Protein expressions related with histologic severity of hepatic steatosis

\begin{tabular}{|c|c|c|c|c|c|c|c|c|c|}
\hline & \multicolumn{3}{|c|}{ pSTAT3 in hepatocytes } & \multicolumn{3}{|c|}{ Estrogen-receptor a in hepatocytes } & \multicolumn{3}{|c|}{ pSTAT3 in hepatic stellate cells } \\
\hline & negative & positive & $P$ value & focal & diffuse & $P$ value & negative & positive & $P$ value \\
\hline \multicolumn{3}{|c|}{ Lobular inflammation (inflammatory foci/ 200× field) } & 0.028 & & & 0.022 & & & 0.016 \\
\hline$<2$ & $16(55 \%)$ & $5(17 \%)$ & & $3(10 \%)$ & $18(62 \%)$ & & $20(69 \%)$ & $1(3 \%)$ & \\
\hline $2-4$ & $2(7 \%)$ & $4(14 \%)$ & & $2(7 \%)$ & $4(14 \%)$ & & $3(10 \%)$ & $3(10 \%)$ & \\
\hline$>4$ & 0 & $2(7 \%)$ & & $2(7 \%)$ & 0 & & $1(3 \%)$ & $1(3 \%)$ & \\
\hline \multicolumn{2}{|c|}{ Ballooning degeneration } & & 0.010 & & & 0.001 & & & 0.033 \\
\hline none & $10(34 \%)$ & $1(3 \%)$ & & 0 & $11(38 \%)$ & & 11 (38\%) & 0 & \\
\hline few & 7 (24\%) & $5(17 \%)$ & & $2(7 \%)$ & $10(34 \%)$ & & 10 (34\%) & $2(7 \%)$ & \\
\hline many & $1(3 \%)$ & $5(17 \%)$ & & $5(17 \%)$ & $1(3 \%)$ & & $3(10 \%)$ & $3(10 \%)$ & \\
\hline Fibrosis & & & 0.001 & & & 0.000 & & & 0.000 \\
\hline absent & $11(38 \%)$ & 0 & & 0 & $11(38 \%)$ & & $11(38 \%)$ & 0 & \\
\hline mild $^{a}$ & $6(231 \%)$ & $4(14 \%)$ & & 0 & $10(34 \%)$ & & 10 (34\%) & 0 & \\
\hline advanced $^{b}$ & $1(3 \%)$ & $7(24 \%)$ & & 7 (24\%) & $1(3 \%)$ & & $3(10 \%)$ & $5(17 \%)$ & \\
\hline
\end{tabular}




\section{Discussion}

The present study identifies PSTAT3 as a potential marker of histologic severity in hepatic steatosis. pSTAT3 may be required in disease progression of hepatic steatosis. Our data showed that pSTAT3 expression in hepatocytes and hepatic stellate cells correlated significantly with severe lobular inflammation, severe ballooning degeneration, and advanced fibrosis. These pSTAT3 results are in agreement with previous animal and cell culture studies in which interleukin- 6 promoted liver inflammation by activating hepatic STAT3 [30]. To the best of our knowledge, this is the first report of pSTAT3 expression in the liver tissue of hepatic steatosis patients.

Previous studies also found that STAT3 activation in hepatocytes and hepatic stellate cells lead to hepatic fibrosis [31-35]. Here, STAT3 mediated the effects of leptin via collagen gene activation in a liver fibrogenesis mouse model [31, 32]. STAT3 may promote hepatic fibrosis $[36,37]$ through the upregulation of tissue inhibitor of metalloproteinases-1 [33, 34] and transforming factor- $\beta$ expression [37]. On the other hand, repression of STAT3 expression was found to exacerbate liver inflammation in interleukin-10-deficient mice [38] and accelerate hepatic fibrosis during cholestasis [39]. Further study will be required to determine if there might be condition-specific feedback loops that enhance or inhibit STAT3 function.

In hepatic steatosis with severe or advanced histomorphologic features, we found estrogen-receptor $\alpha$ expression was focal rather than diffuse. Our data suggest that diffuse expression of estrogen-receptor $\alpha$ may actually have a protective effect against disease progression, potentially by serving as a hepatic receptor for estradiol [40], which inhibits the generation of reactive oxygen species and suppresses hepatic stellate cell activation, resulting in reduced proliferation and collagen production [13, 41]. Protective effects of estrogen in patients with hepatic fibrosis have been reported by others [41]. Moreover, an estradiol study where mice were administered a high-fat diet plus ethanol suggested that an estrogen conjugate might benefit both NAFLD and AFLD patients [42]. However, reports showing the expression patterns of estrogen-receptor $\alpha$ in human hepatic nuclei are rare [43].

Our data indicates that both pERK in hepatic stellate cells and estrogen-receptor $\alpha$ in hepatocytes may be linked to the development of hepatic steatosis. pERK was expressed in hepatic stellate cells in all hepatic steatosis cases examined. Previous studies of rodent liver tissue also implicate pERK in the development of hepatic steatosis, as well as steatohepatitis [44-46]. Moreover, pERK signaling in activated hepatic stellate cells was pro-fibrogenic in NAFLD [15] and AFLD [47] patients in previous studies. Estrogen-receptor $\alpha$ was sufficiently expressed in all NAFLD and AFLD cases. In chicken primary hepatocytes, estrogen enhanced hepatic fatty acid synthesis [48]. In NAFLD patients with elevated serum estrogen levels [11], estrogen entered hepatocytes, resulting in the translocation of ligand-bound estrogenreceptors to the nucleus [49]. Serum estrogen levels are known to be elevated in alcoholic men [50], perhaps owing, at least in part, to the presence of biologically active phytoestrogen (derived from plant-based ingredients such as grains, fruits, and hops) in alcoholic beverages [51].

To our knowledge, this is the first report of mTOR nuclear expression in human liver tissue. mTOR was previously believed to localize exclusively to the cytoplasm; however, recent reports document its presence in multiple intracellular compartments, including the nucleus, as well as shuttling between the cytoplasm and nucleus. However, a specific nuclear function for mTOR has yet to be established [51-53].

This retrospective study does have some limitations. First, many of the NAFLD patients were young men at lessadvanced disease stage. This reflects the inclusion of men who underwent medical check-ups prior to mandatory military service in Korea; our hospital is among the institutions providing official health documentation. Second, it was not determined whether mTOR, STAT3 and ERK belong to leptin signaling pathways. Leptin and leptinreceptor antibodies used in this study did not specifically recognize their target antigens in human liver tissue. This occurred despite previous use of these antibodies to specifically detect leptin and leptin-receptor in the hepatocellular carcinoma (Additional file 2), breast, biliary tract, appendix and stomach $[54,55]$.

\section{Conclusions}

Here we have shown that pSTAT3 expression correlates with severe histomorphologic features in hepatic steatosis, and suggest that diffuse expression of estrogenreceptor $\alpha$ may lessen severity. Hence, both proteins may play a role in mechanisms of disease progression in patients with hepatic steatosis. Our results also implicate pERK and estrogen-receptor $\alpha$ in the development of hepatic steatosis. How these proteins modulate the disease development process is, however, unclear and warrants further study.

\section{Additional files}

Additional file 1: Differential features between non-alcoholic fatty liver disease (NAFLD) subgroups stratified by NAS and SAF. (DOCX $47 \mathrm{~kb}$ )

Additional file 2: Immunohistochemical staining for leptin (A-20, 1:50; Santa Cruz Biotechnology, Santa Cruz, CA, USA) and leptin-receptor (B-3, 1:25; Santa Cruz Biotechnology) in hepatic steatosis cases, and non-neoplastic hepatocytes and carcinoma cells from hepatocellular carcinomas. (a-b) Hepatic steatosis with nuclear and/or cytoplasmic staining of leptin (a), and diffuse granular staining of leptin-receptor (b). (c-e) Leptin in hepatocellular carcinomas. Nuclear and cytoplasmic staining of leptin in non-neoplastic hepatocytes (left side of each picture), and negative (c), weak (d) or strong (e) leptin staining in hepatocellular 
carcinoma cells (right side of each picture). (f-g) Leptin-receptor in hepatocellular carcinomas. Diffuse granular cytoplasmic expression of leptin-receptor in non-neoplastic hepatocytes (left), and weak focal (f) or strong diffuse (g) staining of leptin-receptor in hepatocellular carcinoma cells (right). (JPEG $4962 \mathrm{~kb}$ )

\section{Abbreviations}

AFLD: Alcoholic fatty liver disease; ALT: Alanine transaminase; BMI: Body mass index; mTOR: Mammalian target of rapamycin; NAFLD: Non-alcoholic fatty liver disease; NASH CRN NAS: Non-alcoholic Steatohepatitis Clinical Research Network NAFLD activity score; pERK: Phosphorylated extracellular signal-regulated kinase; PSTAT: Phosphorylated signal transducer and activator of transcription; SAF: Steatosis, activity, and fibrosis

\section{Acknowledgments}

We thank Jin Hee Han, B.S. for technical assistance in performing the immunohistochemistry.

\section{Funding}

This work was supported by the Basic Science Research Program of the National Research Foundation of Korea, which is funded by the Ministry of Education (2016R1D1A1B01010316).

\section{Availability of data and materials}

Please contact the corresponding author for data requests.

\section{Authors' contributions}

EC reviewed the histomorphologic and immunohistochemical data, carried out the statistical analysis, and helped draft the manuscript. WK analyzed the clinical data and helped draft the manuscript. SKJ collected the clinical data. SP reviewed the histomorphologic and immunohistochemical data. JHP contributed to the statistical analysis and histomorphologic review. YKK reviewed the histomorphologic and immunohistochemical data and the pertinent literature S-YJ aided in the histomorphologic review. MSC conceived, designed, and coordinated the study, analyzed the histomorphologic and immunohistochemical data, performed the statistical analysis, provided financial support, and helped draft the manuscript. All authors read and approved the final manuscript.

\section{Ethics approval and consent to participate}

All human liver tissue specimens were obtained during diagnostic and therapeutic procedures. Consent to participate was not required because this was a retrospective study of formalin-fixed, paraffin-embedded tissues obtained after pathological diagnosis. All samples were anonymized before initiating the study. The retrospective study protocol was reviewed and approved by the Institutional Review Board of the Seoul National University Boramae Hospital with the condition of anonymization (IRB No. 26-2016-12/022). The study was conducted in accordance with the principles of the Declaration of Helsinki.

\section{Consent for publication}

Not applicable

\section{Competing interests}

The authors declare no conflicts of interest.

\section{Publisher's Note}

Springer Nature remains neutral with regard to jurisdictional claims in published maps and institutional affiliations.

\section{Author details}

'Department of Pathology, Seoul National University Boramae Hospital, Seoul National University College of Medicine, 20 Boramae-ro 5-gil, Dongjak-gu, Seoul 07061, Korea. ${ }^{2}$ Department of Internal Medicine, Seoul National University Boramae Hospital, Seoul National University College of Medicine, 20 Boramae-ro 5-gil, Dongjak-gu, Seoul, Korea. ${ }^{3}$ Department of Pathology, Seoul Paik Hospital, Inje University College of Medicine, Mareunnae-ro 9, Jung-gu, Seoul, Korea. ${ }^{4}$ Department of Pathology, Soon Chun Hyang University Hospital, 59 daesagwan-ro, Yongsan-gu, Seoul, Korea.
Received: 5 December 2017 Accepted: 12 March 2018

Published online: 03 April 2018

\section{References}

1. Naran NH, Haagensen M, Crowther NJ. Steatosis in South African women: how much and why? PLoS One. 2018:13:e0191388.

2. Powell EE, Jonsson JR, Clouston AD. Steatosis: co-factor in other liver diseases. Hepatology. 2005;42:5-13.

3. Laplante M, Sabatini DM. An emerging role of mTOR in lipid biosynthesis. Curr Biol. 2009;19:R1046-52.

4. Sozio MS, Liangpunsakul S, Crabb D. The role of lipid metabolism in the pathogenesis of alcoholic and non-alcoholic hepatic steatosis. Semin Liver Dis. 2010:30:378-90.

5. Kubrusly MS, Corrêa-Giannella ML, Bellodi-Privato M, de Sá SV, de Oliveira CP, Soares IC, et al. A role for mammalian target of rapamycin (mTOR) pathway in non-alcoholic steatohepatitis related-cirrhosis. Histol Histopathol. 2010;25:1123-31.

6. Elinav E, Ali M, Bruck R, Brazowski E, Phillips A, Shapira Y, et al. Competitive inhibition of leptin signaling results in amelioration of liver fibrosis through modulation of stellate cell function. Hepatology. 2009:49:278-6.

7. Maya-Monteiro CM, Bozza PT. Leptin and mTOR: partners in metabolism and inflammation. Cell Cycle. 2008;7:1713-7.

8. Saxena NK, Saliba FJJ, Anania FA. Leptin induced increased a2(I) collagen gene expression in cultured rat hepatic stellate cells. J Cell Biochem. 2003; 89:311-20.

9. Kagan P, Sultan M, Tachlytski I, Safran M, Ben-Ari Z. Both MAPK and STAT3 signal transduction pathways are necessary for IL-6-dependent hepatic stellate cells activation. PLoS One. 2017;12:e0176173.

10. Svegliati-Baroni G, Ridolfi F, Caradonna Z, Alvaro D, Marzioni M, Saccomanno $S$, et al. Regulation of ERK/JNK/p70S6K in two rat models of liver injury and fibrosis. J Hepatol. 2003;39:528-37.

11. Mintziori G, Poulakos P, Tsametis C, Goulis DG. Hypogonadism and nonalcoholic fatty liver disease. Minerva Endocrinol. 2017;42:145-50

12. Uebi T, Umeda M, Imai T. Estrogen induces estrogen-receptor alpha expression and hepatocyte proliferation in the livers of male mice. Genes Cells. 2015;20:217-23.

13. Itagaki T, Shimizu I, Cheng X, Yuan Y, Oshio A, Tamaki K, et al. Opposing effects of oestradiol and progesterone on intracellular pathways and activation processes in the oxidative stress induced activation of cultured rat hepatic stellate cells. Gut. 2005;54:1782-9.

14. Moreira RK. Hepatic stellate cells and liver fibrosis. Arch Pathol Lab Med. 2007:131:1728-34

15. Zhang F, Zhang Z, Kong D, Zhang X, Chen L, Zhu X, et al. Tetramethylpyrazine reduces glucose and insulin-induced activation of hepatic stellate cells by inhibiting insulin receptor-mediated PI3K/AKT and pERK pathways. Mol Cell Endocrinol. 2014;382:197-204.

16. Friedman SL. Molecular regulation of hepatic fibrosis, an integrated cellular response to tissue injury. J Biol Chem. 2000;275:2247-50.

17. Hellerbrand C. Pathophysiological similarities and synergisms in alcoholic and non-alcoholic steatohepatitis. Dig Dis. 2010;28:783-91.

18. Spahr L, Hadengue A. Alcoholic and non-alcoholic steatohepatitis: the same disease! II Management Rev Med Suisse. 2005;1:2032-4.

19. Day CP, James OF. Steatohepatitis: a tale of two "hits"? Gastroenterology. 1998:114:842-5

20. Yang $S Q$, Lin $H Z$, Lane MD, Clemens $M$, Diehl AM. Obesity increases sensitivity to endotoxin liver injury: implications for the pathogenesis of steatohepatitis. Proc Natl Acad Sci U S A. 1997;94:2557-62.

21. Kojima H, Sakurai S, Uemura M, Takekawa T, Morimoto H, Tamagawa Y, Fukui H. Difference and similarity between non-alcoholic steatohepatitis and alcoholic liver disease. Alcohol Clin Exp Res. 2005:29:259S-63S.

22. Diehl AM, Goodman Z, Ishak KG. Alcohol like liver disease in non-alcoholics. A clinical and histologic comparison with alcohol-induced liver injury. Gastroenterology. 1988;95:1056-62.

23. Yip WW, Burt AD. Alcoholic liver disease. Semin Diagn Pathol. 2006;2:149-60.

24. Arteel GE. Oxidants and antioxidants in alcohol-induced liver disease. Gastroenterology. 2003;124:778-90

25. Brunt EM, Janney CG, Di Bisceglie AM, Neuschwander-Tetri BA, Bacon BR. Non-alcoholic steatohepatitis: a proposal for grading and staging the histological lesions. Am J Gastroenterol. 1999:94:2467-4.

26. Kleiner DE, Brunt EM, Van Natta M, Behling C, Contos MJ, Cummings OW, et al. Design and validation of a histological scoring system for non-alcoholic fatty liver disease. Hepatology. 2005;41:1313-21. 
27. Steering Committee of the Western Pacific Region of the World Health Organization, the International Association for the Study of Obesity, and the International Obesity Task Force. The Asia-Pacific perspective: redefining obesity and its treatment. Melbourne: Health Communications Australia Pty Ltd; 2000. p. 8-56.

28. Bedossa P. Utility and appropriateness of the fatty liver inhibition of progression (FLIP) algorithm and steatosis, activity, and fibrosis (SAF) score in the evaluation of biopsies of non-alcoholic fatty liver disease. Hepatology. 2014;60:565-75.

29. Bedossa P, Poitou C, Veyrie N, Bouillot JL, Basdevant A, Paradis V, et al. Histopathological algorithm and scoring system for evaluation of liver lesions in morbidly obese patients. Hepatology. 2012;56:1751-9.

30. He G, Karin M. NF-kappaB and STAT3-key players in liver inflammation and cancer. Cell Res. 2011:21:159-68.

31. Zhang W, Niu M, Yan K, Zhai X, Zhou Q, Zhang L, Zhou Y. Stat3 pathway correlates with the roles of leptin in mouse liver fibrosis and sterol regulatory element binding protein-1c expression of rat hepatic stellate cells. Int J Biochem Cell Biol. 2013;45:736-44.

32. Saxena NK, Ikeda K, Rockey DC, Friedman SL, Anania FA. Leptin in hepatic fibrosis: evidence for increased collagen production in stellate cells and lean littermates of Ob/Ob mice. Hepatology. 2002;35:762-71.

33. Cao Q, Mark KM, Ren C, Lieber CS. Leptin stimulates tissue inhibitor of metalloproteinase-1 in human hepatic stellate cells: respective roles of the JAK/STAT and JAK-medicated $\mathrm{H}_{2} \mathrm{O}_{2}$-dependent MAPK pathways. J Biol Chem. 2004;279:4292-304.

34. Wang $H$, Lafdil F, Wang L, Yin S, Feng D, Gao B. Tissue inhibitor of metalloproteinase 1 (TIMP-1) deficiency exacerbates carbon tetrachlorideinduced liver injury and fibrosis in mice: involvement of hepatocyte STAT3 in TIMP-1 production. Cell Biosci. 2011;1:14.

35. Kong $X$, Horiguchi N, Mori M, Gao B. Cytokines and STATs in liver fibrosis. Front Physiol. 2012;3:69.

36. Ogata H, Chinen T, Yoshida T, Kinjyo I, Takaesu G, Shiraishi H, et al. Loss of SOCS3 in the liver promotes fibrosis by enhancing STAT3-mediated TGF-beta1 production. Oncogene. 2006;25:2520-30.

37. Tang L-Y, Heller M, Meng Z, Yu L-R, Tang Y, Zhou M, et al. Transforming growth factor- $\beta$ (TGF- $\beta$ ) directly activates the JAK1-STAT3 axis to induce hepatic fibrosis in coordination with the SMAD pathway. J Biol Chem. 2017;292:4302-12.

38. Miller AM, Wang H, Bertola A, Park O, Horiguchi N, Ki SH, et al. Inflammation-associated interleukin-6/signal transducer and activator of transcription 3 activation ameliorates alcoholic and non-alcoholic fatty liver diseases in interleukin-10-deficient mice. Hepatology. 2011;54:846-56.

39. Shigekawa M, Takehara T, Kodama T, Hikita H, Shimizu S, Li W, et al. Involvement of STAT3-regulated hepatic soluble factors in attenuation of stellate cell activity and liver fibrogenesis in mice. Biochem Biophys Res Commun. 2011;406:614-20

40. Shimizu I. Impact of oestrogens on the progression of liver disease. Liver Int. 2003;23:63-9.

41. Yasuda M, Shimizu I, Shibata M, Ito S. Suppressive effects of estradiol on dimethynitrosamine-induced fibrosis of the liver in rats. Hepatology. 1999; 29:719-27.

42. Holcomb VB, Hong J, Núñez NP. Exogenous estrogen protects mice from the consequences of obesity and alcohol. Menopause. 2012;19:680-90.

43. Erkan G, Yilmaz G, Konca Degertekin C, Akyol G, Ozenirler S. Presence and extent of estrogen-receptor-alpha expression in patients with simple steatosis and NASH. Pathol Res Pract. 2013;209:429-32.

44. Bai $X$, Hong W, Cai P, Chen Y, Xu C, Cao D, et al. Valproate induced hepatic steatosis by enhanced fatty acid uptake and triglyceride synthesis. Toxicol Appl Pharmacol. 2017;324:12-25.

45. Mahli A, Saugspier M, Koch A, Sommer J, Dietrich P, Lee S, et al. pERK activation and autophagy impairment are central mediators of irinotecaninduced steatohepatitis. Gut. 2017; https://doi.org/10.1136/gutjnl-2016312485. [Epub ahead of print]

46. Wang X, Zhang ZF, Zheng GH, Wang AM, Sun CH, Qin SP, et al. Attenuation of hepatic steatosis by purple sweet potato colour is associated with blocking Src/pERK/C/EBPß signalling in high-fat-diet-treated mice. Appl Physiol Nutr Metab. 2017;42:1082-91.

47. Li J, Hu W, Baldassare JJ, Bora PS, Chen S, Poulos JE, et al. The ethanol metabolite, linolenic acid ethyl ester, stimulates mitogen-activated protein kinase and cyclin signaling in hepatic stellate cells. Life Sci. 2003;73:1083-96.

48. Zhang M, Li CC, Li F, Li H, Liu XJ, Loor JJ, et al. Estrogen promotes hepatic synthesis of long-chain polyunsaturated fatty acids by regulating ELOVL5 at post-transcriptional level in laying hens. Int J Mol Sci. 2017;18. pii: E1405.
49. Gavaler JS. Alcoholic beverages as a source of estrogens. Alcohol Health Res World. 1998;22:220-7.

50. Eagon PK. Alcoholic liver injury: influence of gender and hormones. World J Gastroenterol. 2010;16:1377-84.

51. Rosner $M$, Hengstschläger M. Cytoplasmic and nuclear distribution of the protein complexes mTORC1 and MTORC2: rapamycin triggers dephosphorylation and delocalization of the MTORC2 components rictor and sin1. Hum Mol Genet. 2008;17:2934-48.

52. Tsang CK, Liu H, Zheng XF. mTOR binds to the promoters of RNA polymerase I- and III-transcribed genes. Cell Cycle. 2010;9:953-7.

53. Betz C, Hall MN. Where is mTOR and what is it doing there? J Cell Biol. 2013;203:563-74.

54. Chang MS, Byeon SJ, Yoon SO, Kim BH, Lee HS, Kang GH, et al. Leptin, MUC2 and mTOR in appendiceal mucinous neoplasms. Pathobiology. 2012; 79:45-53.

55. Choi E, Byeon SJ, Kim SH, Lee HJ, Kwon HJ, Ahn H, et al. Implication of leptin-signaling proteins and Epstein-Barr virus in gastric carcinomas. PLoS One. 2015:10:e0130839.

\section{Submit your next manuscript to BioMed Central and we will help you at every step:}

- We accept pre-submission inquiries

- Our selector tool helps you to find the most relevant journal

- We provide round the clock customer support

- Convenient online submission

- Thorough peer review

- Inclusion in PubMed and all major indexing services

- Maximum visibility for your research

Submit your manuscript at www.biomedcentral.com/submit
) Biomed Central 